\title{
Influence of Prosodic Information on the Processing of Split Particles: ERP Evidence from Spoken German
}

\author{
Frédéric Isel $^{1}$, Kai Alter ${ }^{2}$, and Angela D. Friederici ${ }^{1}$
}

\begin{abstract}
Spoken language comprehension involves the use of different sources of linguistic information such as prosodic, syntactic, lexical, and semantic information. The question, however, of "when" and "how" these sources of information are exploited by the language processing system still remains unanswered. In the present study, we used event-related brain potentials (ERPs) to investigate the interaction between prosodic, syntactic, and lexical information during the processing of spoken German sentences. The sentence structure was manipulated by positioning a split particle at the end of the sentences after the occurrence of inflected verb whose lexical entry does not contain a split particle (e.g., *Sie alarmierte den Detektiv an [*She alerted at the detective]) [According to linguistic convention, incorrect sentences are marked by an asterisk.]. The prosodic contour of the verb stems was manipulated such that it marked either the presence of a split particle at a later position in the sentence or not. Participants performed an off-line probe-detection task. ERP data indicate
\end{abstract}

\section{INTRODUCTION}

In the present study, a combined behavioral-electrophysiological method was employed to study whether the parser exploits prosodic information in building up phrase structure representations. In particular, we approached this issue by investigating whether the intonation of German-inflected verb stems (e.g., laechelte [smiled]) assists the parser to "predict" the later occurrence of a split particle (e.g., an [at]). ${ }^{1}$

One central distinction between psycholinguistic models of language comprehension involves the autonomy versus the interdependence of stages of processing. On the one hand, the so-called serial models (e.g., Gorrell, 1995; Frazier, 1987) claim that the parser initially builds up a syntactic structure independent of lexical-semantic or sentential-semantic information. On the other hand, the so-called interactive models (e.g., McClelland, St. John, \& Taraban, 1989; Marslen-Wilson \& Tyler, 1980) propose that structural and lexical-semantic information interact continuously during parsing, allowing ini-

\footnotetext{
${ }^{1}$ Max Planck Institute for Human Cognitive and Brain Sciences, Leipzig, Germany, ${ }^{2}$ University of Newcastle, Newcastle, UK
}

that prosodic information of German-inflected verb stems is consulted on-line by the language processing system ("parser") in order to "predict" the presence of a split particle at a later position in the sentence. An N400 effect was observed for the processing of split particles following verb stems which do not take a particle. However, this effect was only observed when the prosody of the verb stem did signal the presence of a split particle. We argue that the N400 component reflects the high costs associated with the lexical search that the language processing system has to perform when confronted with nonexisting words such as these resulting from the combination of the split particle and the verb stem in the present study. Furthermore, as a general reflection of prosodic processes, a Closure Positive Shift (CPS) was found at intonational phrase boundaries. In sum, the present findings provide strong evidence that prosodic information is a good "predictor" of upcoming information during the auditory processing of German sentences.

tial structure building processes to be influenced by lexical knowledge. However, none of these models explicitly discusses the role of prosody on syntactic analysis, presumably as models are based on reaction-time measurements taken during the reading of sentences. Nevertheless, the integration of prosody into psycholinguistic models explicitly called for by Fodor (2002) can no longer be escaped as there is accumulating evidence that prosodic information can affect syntactic processes both from behavioral studies (see Cutler, Dahan, \& van Donselaar, 1997, for a review) and also from a recent event-related brain potential (ERP) study (Steinhauer, Alter, \& Friederici, 1999).

Friederici (2002) formulated a neurochronometric model on the basis of combined ERP data obtained in different languages in which the interaction between prosodic and syntactic information is considered. However, temporal structure of this interaction is not yet specified. In contrast, recent neuroanatomical evidence suggests that the observed interaction between prosodic and syntactic information might be attributed to dynamic interaction between the left and the right hemispheres. Precisely, this interaction is assumed to be dependent on the corpus callosum, interconnecting 
the two hemispheres. On the basis of combined neuroanatomical data, Friederici and Alter (in press) formulated the dynamic dual pathway model. However, the next generation of neurocognitive parsing models will have to clearly specify the temporal structure of the interaction between prosodic information and syntactic analysis. Here we investigated this interaction using event-related potentials (ERPs).

Converging behavioral findings support the view that prosody can guide the computation of a correct phrase structure assignment in ambiguous sentences that can admit alternative syntactic analyses. Studies using sentences with a global surface structure ambiguity that admit more than one syntactic (and semantic) interpretation (e.g., I read about the repayment with interestdoes the prepositional phrase with interest refer to my reading or to the repayment?) showed that listeners are able to correctly infer the intended interpretation of the sentence using the prosodic correlates of syntactic boundaries (i.e., durational information) - that is, preboundary lengthening as well as pitch-contour variation, usually a preboundary fall-rise or rise ${ }^{2}$ (Ferreira, Anes, \& Horine, 1996; Price, Ostendorf, ShattuckHufhagel, \& Fong, 1991; Lehiste 1972, 1973). However. although these studies suggest a close relationship between prosody and syntax, they did not address the issue of "when" and "how" prosodic information is used by the parser. The only way to approach this issue is by using more on-line tasks such as, for example, the cross-modal priming technique (Nicol \& Pickering, 1993). Studies using temporary closure ambiguity either in complete sentences (Nagel, Shapiro, Tuller, \& Nawy, 1996; Speer, Kjelgaard, \& Dobroth, 1996), or in sentence fragments (Warren, Grabe, \& Nolan, 1995; Marslen-Wilson, Tyler, Warren, Grenier, \& Lee, 1992; Beach, 1991) are more suitable for specifying the time course of syntactic disambiguation, as the point in the sentence at which the ambiguity is resolved is precisely identifiable. Consider a sentence such as "The workers considered the last offer from the management was a real insult" (Holmes, Kennedy, \& Murray, 1987). This sentence is locally ambiguous in its structure because the noun phrase which follows the verb (e.g., "the last offer from the management") can be either the direct object of the preceding verb (e.g., "considered"), or the subject of a subsequent clause-complement (e.g., "the last offer from the management was ..."). MarslenWilson, Tyler, Warren, et al. (1992), using the on-line cross-naming task with similar sentence material, showed that prosodic information of the temporarily ambiguous portion of the sentence (in the example above, "the last offer from the management") which precedes disambiguating lexical information (e.g., "was"), ${ }^{3}$ are effective in controlling how listeners resolve the attachment ambiguities. Participants heard auditory sentence fragments (i.e., the primes) that either contained the complementizer "that" (+Comp condition: "The workers consid- ered that the last offer from the management") or did not (-Comp condition: "The workers considered the last offer from the management"). In the -Comp condition, the prosody of the nominal phrase "The last offer from the management" favors a strategy of nonminimal attachment (NMA). It means that "The last offer from the management" should be analyzed as the subject phrase of a subsequent clause complement rather than the direct object of the verb "considered." The auditory primes were followed by a visual probe (i.e., the auxiliary WAS). The dependent variables were the latencies to name the probe. The authors hypothesized that the naming latencies of the probe WAS should be longer if the auxiliary WAS is incongruent with the preceding context in term of syntactic attachment. It would be the case if the participants favor a strategy of minimal attachment (MA), that is, they process "The last offer from the management" as the direct object of the verb "considered." In contrast, if participants used an NMA strategy, then the naming latencies should be similar to the latencies found in a control condition (+Comp condition). Despite the omission of the explicit complementizer "that," listeners nonetheless favored a clausecomplement interpretation by using a strategy of NMA: ["The workers considered"] ${ }^{\mathrm{IPh} 1}$ ["the last offer from the management was a real insult" $]^{\mathrm{IPh} 2}$ as indicated by the absence of a lengthening of the naming latencies in the -Comp condition. This evidence suggests that the ambiguous region of the sentence presumably contains the critical prosodic information which allow the parser to select the appropriate syntactic analysis. MarslenWilson and coworkers concluded that prosodic information can be used on-line to resolve potential ambiguities in the structural interpretation of the utterance. However, caution is at order before drawing firm conclusions regarding the exact role of prosody on syntactic parsing. Contradictory findings challenging the view that prosody can immediately influence parsing decisions have also been reported (Pynte \& Prieur 1996; Stirling \& Wales, 1996; Watt \& Murray, 1996). But, as suggested by Steinhauer and Friederici (2001, p. 270), apart from the complex interplay of acoustic parameters, one important reason for the mixed data, and a major drawback in this field, was the much regretted lack of an appropriate on-line method.

Another function of prosody, namely, predictor of utterance length, was revealed by Grosjean and Hirt (1996) and Grosjean (1983) using the gating paradigm. ${ }^{4}$ Unfortunately, the investigation of the predictive power of prosody during sentence comprehension has not received much attention until now. Grosjean and Hirt, and Grosjean showed that English listeners (Experiments 1 and 2) as well as French listeners (Grosjean \& Hirt, 1996; Experiment 3) in processing their native language are able to determine on the basis of prosodic information (relative F0 and syllable duration) whether a sentence is over or not when listening to the potentially 
last word of a sentence. ${ }^{5}$ Unlike French listeners, English listeners are also proficient at estimating how much longer the sentence will continue (3 or 6 words) when it is not over, and this by relying solely on prosodic cues. Grosjean concluded that durational information as well as pitch-contour variation are relevant predictors of what is about to occur in a sentence, at least in English. On the basis of these data, Gee and Grosjean (1983) formulated a model of sentence production including an intonational component.

Finally, recent ERP studies have shed new light on the role of prosody in determining the initial parsing decision. Steinhauer, et al. (1999), using spoken German sentences with temporary syntactic closure ambiguity, showed that the duration and pitch-contour variations measured at intonational phrase boundaries are reflected by a characteristic centro-parietal positive-going waveform. The authors labeled this ERP component the "closure positive shift" (CPS). Steinhauer, et al. (Experiment 3) clearly demonstrated that the CPS reflects the processing of the prosodic boundary as marked by lengthening of the prefinal syllable and a change in the F0 contour rather than the perception of a pause interrupting the speech input. Furthermore, using the technique of cross-splicing, Steinhauer, et al. showed that sentences presenting a mismatch between prosodic information and syntactic constraints elicited an N400-P600 pattern of ERP components, reflecting a prosody-induced garden-path effect. Recently, Steinhauer and Friederici (2001) showed that the CPS is also a reliable marker for prosodic phrasing during reading based on comma rules. ${ }^{6}$ Finally, the prosodic rather syntactic nature of the processes underlying the CPS component was demonstrated by reporting the CPS even in delexicalized sentence melodies (Experiment 3). From their combined ERP data, Steinhauer and Friederici concluded that the CPS appears to be a universal reflection of prosodic phrasing, independent of input modality.

Here we investigated the predictive power prosody can have during the processing of spoken German sentences using on-line ERP measures. Due to the morphological specificities of the German language, we addressed the question of whether prosody is a good predictor of upcoming information via a study on the processing of complex German verbs with a split particle (e.g., an-laecheln [to smile-at]). In German, the particle must be placed at the end of the sentence (e.g., Sie laechelte den Arbeiter an). ${ }^{7}$ Using the crosssplicing technique, we manipulated the prosodic contour of verbs whose lexical entry does not contain a split particle (e.g., nennen [to call]) such that the parser should expect a split particle or not. We also manipulated the sentence structure by positioning a split particle at the end of the sentence after verbs usually having no split particles coded in their lexical entry (e.g., *Se nannte den Namen an [*She called at the name]). We thus created a local morpholexical violation.

We addressed the foregoing issues using the experimental paradigm illustrated in Table 1. ERPs were recorded from 20 participants while they were processing correct or morpholexically and/or prosodically incorrect German sentences. The critical item, to which the ERPs were measured, was the split particle or the preposition italicized for clarity in Table 1 . At the acoustic offset of each sentence, participants performed an off-line probedetection task (PDT), that is, they had to judge whether a visually presented word was heard in the preceding sentence. Only correctly answered trials were kept for later statistical analyses of behavioral and ERP data. We compared each condition against the Correct 1 condition which served as control. We explored the effect of prosodic information in predicting the presence of a particle at a later position of the sentence by comparing the ERP responses to the processing of the split particles when the prosodic contour of the verb stem marks the presence of a split particle (Morpholexical condition) with the ERP responses when the prosodic contour of the verb stem does not mark the presence of split particle (Prosodic 1 condition).

On the basis of the neurochronometric model proposed by Friederici (1995, 2002) as well as on previous electrophysiological data in German (Steinhauer, et al., 1999), the following predictions were formulated. In the Correct 1 condition, a CPS should be found at the critical item (i.e., the split particle) after the first full nounphrase (NP) because it is located at an intonational phrase boundary. In both the Correct 2 and 3 conditions, in contrast, no CPS should be elicited at the critical item (i.e., the preposition) after the first full $\mathrm{NP}$ as it does not mark a prosodic boundary. In the Morpholexical condition, the verb stems do not take a split particle. However, the prosodic contour of the verb stem was manipulated in a way that it does signal the presence of a split particle. Thus, if the prosodic contour of the verb stem assists the processor to predict the presence of a particle, then the processing of a particle in the Morpholexical condition should elicit an N400 effect. The N400 should reflect the unsuccessful mapping of the prosodically predicted particle together with the verb stem onto a representation in the mental lexicon. Moreover, a P600 component might possibly be expected, if in addition to a morpholexical mismatch, syntactic repair is triggered. In contrast, no such biphasic N400-P600 pattern should be observed in the Prosodic 1 condition as the prosodic contour of the verb stem does not mark the presence of a split particle. Thus, no attempt to combine the split particle with the verb stem should be engaged. Alternatively, if the prosodic contour of the verb stem is not determinant in predicting upcoming information, no ERP effect should be expected in both Morpholexical and Prosodic 1 conditions. Finally, if the signal manipulation at the 
Table 1. Example of Control and Experimental Sentences with English Translation, and Durations (msec) of the Inflected Verbs, Split Particles, and Prepositional Phrases

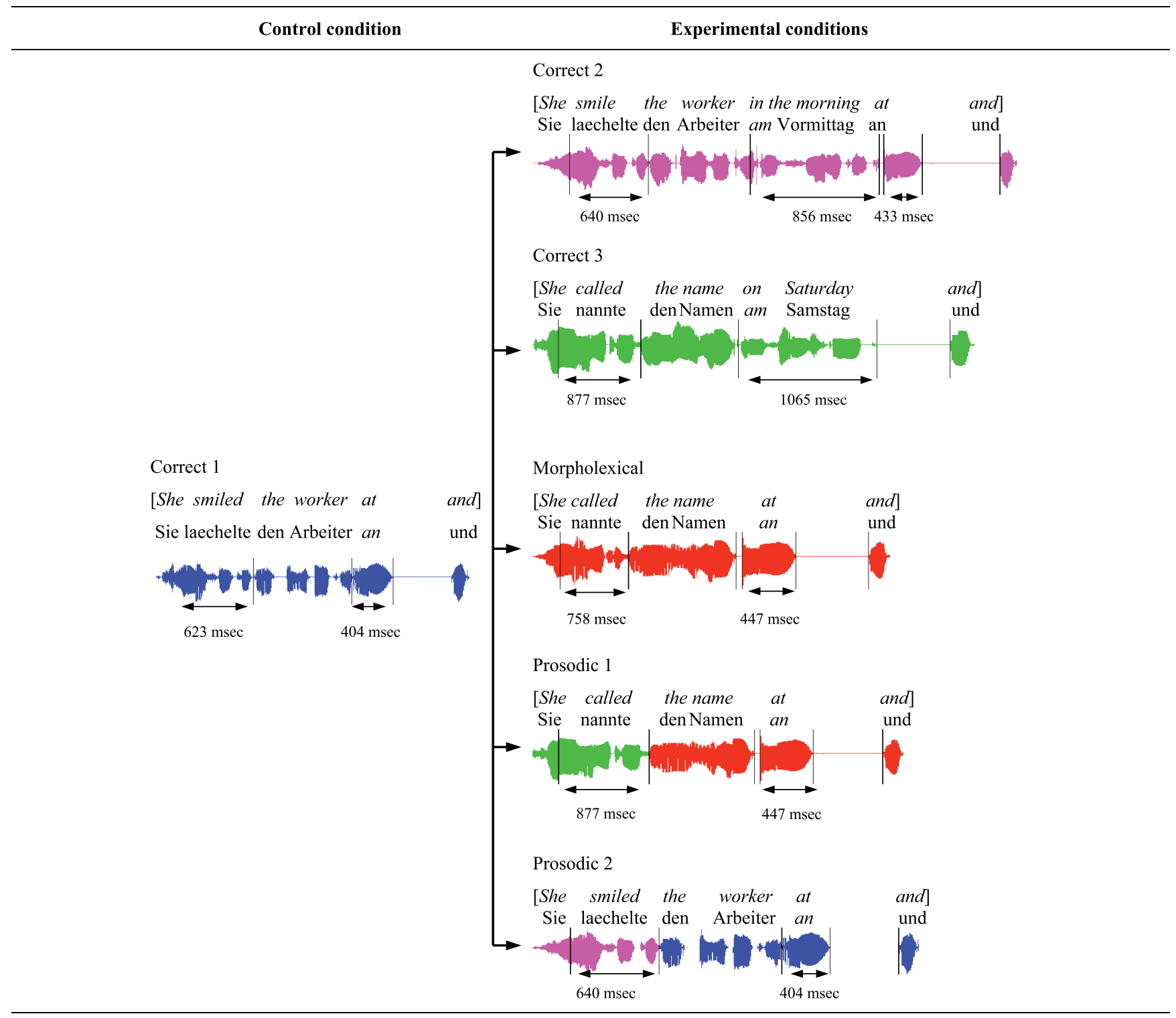

Prosodic 1 and 2 conditions were cross-spliced. Colors indicate from which conditions the different parts of the speech were taken.

splicing point was correctly performed in our study, no ERP effect should be found for the processing of the cross-spliced sentences in the Prosodic 2 condition.

\section{RESULTS}

\section{Behavioral Data}

The mean percentages of errors and the mean verification latencies to accurate responses are given in Table 2. The analysis of variance (ANOVA) conducted on the percentage of errors failed to show a significant main effect of condition. By contrast, the ANOVA run on the mean verification latencies indicated a significant main effect of condition $\left[F_{1}(5,95)=13.27, p<.01, M S E=\right.$ 335.4]. Subsequent post hoc tests indicated that latencies were significantly slower in the Correct 3 , Prosodic 1 , and Morpholexical conditions than in the Correct 1, Correct 2, and Prosodic 2 conditions. Whereas both the Correct 3 and the Morpholexical conditions did not differ from the Prosodic 1 condition, mean verification latencies in the Correct 3 condition were significantly slower than in the Morpholexical condition. Finally, Correct 1, Correct 2, and Prosodic 2 conditions did not significantly differ.

In summary, the PDT was carried out very well (overall error rate $=2.2 \%$ ), indicating that participants were carefully processed the auditory presented sentences. 
Table 2. Behavioral Data

\begin{tabular}{lcc}
\hline Condition & \% Error & Latency \\
\hline Correct 1 & $2.3(0.6)$ & $682(23.5)$ \\
Correct 2 & $1.2(0.5)$ & $681(23.3)$ \\
Correct 3 & $3.0(0.7)$ & $716(25.3)$ \\
Morpholexical & $3.9(0.9)$ & $700(24.6)$ \\
Prosodic 1 & $1.6(0.6)$ & $707(24.6)$ \\
Prosodic 2 & $1.3(0.4)$ & $683(25.3)$ \\
\hline
\end{tabular}

Mean percentages of errors and mean verification latencies to accurate responses in milliseconds (standard errors of the means are in parentheses)

However, the delay of verification latencies observed in the Correct 3, the Morpholexical, and the Prosodic 1 conditions suggests that participants had more difficulties carrying out the task in these experimental conditions. We propose that the longer verification latencies observed in Morpholexical as well as in Prosodic 1 conditions could be due to a disruption of the decisionmaking process caused by the presence of a phoneticgraphemic mismatch between the verb forms present in the auditory sentences and the verb forms used as visual probes. In these conditions, participants heard verbparticle pairs such as nannte an (novel verb, nonexisting in the German lexicon, which we called "pseudoverb" throughout this article), and then saw visual probes such as nannte (word in German). The imperfect match between the word-probe and the spoken pseudoverb due to the presence of the split particle (i.e., an) could have disturbed the participants. As consequence, participants might have needed more time for giving a "yes" response. Furthermore, to account for the longer latencies reported in the Correct 3 condition, we considered the following explanation in terms of memory load. Post hoc acoustic measures performed on the speech signals showed that mean duration of the prepositional phrase is significantly longer in the Correct 3 (1065 msec, SEM = 22.2) than in the Correct 2 condition $(856 \mathrm{msec}, S E M=$ $18.5, p<.01$; see Table 1$)$. Thus, it appears that the longer participants had to keep information in memory, for example, in order to process the long sentences in the Correct 3 condition, the more difficult it becomes to verify whether a visual probe presented at the acoustic offset of the sentence was or was not part of them. This difficulty could be reflected by a lengthening of the verification latencies.

\section{Event-related Potentials}

The waveforms of averaged ERPs are displayed for each of the five conditions separately evaluated against the Correct 1 condition in Figure 1. The visual inspection of these waveforms revealed the following pattern. In the Correct 1 condition, a CPS should be found at the critical item (i.e., the split particle) after the first full $\mathrm{NP}$ as it is located at an intonational phrase boundary. In both the Correct 2 and 3 conditions, in contrast, no CPS should be elicited at the critical item (i.e., the preposition) after the first full NP as it does not mark a prosodic boundary.

Whereas Correct 1 sentences elicited a CPS peaking around 1100 msec after the acoustic onset of the particles (i.e., the critical item), no CPS was elicited at the preposition (i.e., the critical item) in the Correct 2 and Correct 3 conditions. A negativity peaking around 400 msec after the acoustic onset of the particles (N400), and largely distributed over all electrode sites except the midline electrodes ( $\mathrm{Fz}, \mathrm{Cz}, \mathrm{Pz})$, was observed for the processing of morpholexically incorrect sentences containing inflected verbs that do not take a particle but whose prosodic contour marked the presence of a split particle at a later position of the sentence (Morpholexical condition). In contrast, the processing of the same morpholexically incorrect sentences containing inflected verbs, whose prosodic contour did not mark the presence of a split particle at a later position of the sentence (Prosodic 1 condition), did not elicit an N400 component. Finally, the Prosodic 2 condition failed to show significant ERP effects.

The statistical analyses of the three-way ANOVAs with the variables Condition ( 6 levels) $\times$ Region (anterior, posterior $) \times$ Hemisphere (left, right) were calculated for the six conditions for the four following time windows: 150-280, 300-500, 700-1000, and 1000-1500 msec. ${ }^{8}$

The analysis for the time window 150-280 msec failed to reveal either significant main effects of condition, or significant Condition $\times$ Region or Condition $\times$ Hemisphere interactions. Similarly, the ANOVA performed on the midline electrodes did not indicate significant ERP effects.

The ANOVA conducted for the time window 300$500 \mathrm{msec}$ revealed that the Morpholexical condition was significantly different from the Correct 1 condition $[1 \mathrm{C} ; F(1,19)=7.28, p<.05, M S E=3.3]$. The absence of a Condition $\times$ Region $(F<1)$, or a Condition $\times$ Hemisphere $[F(1,19)=1.35, p>.10, M S E=0.3]$ interaction reflects a wide distribution of the N400 component over all electrode sites in the Morpholexical condition (1C). Moreover, the ANOVA performed on the three midline electrodes only revealed a marginally significant main effect of condition $[F(1,19)=3.30, p<.10, M S E=7.5]$.

In contrast, the Prosodic 1 condition (1d) did not significantly differ from the Correct 1 condition $[F(1,19)=$ $2.31, p>.10, M S E=8.4]$. Moreover, neither a significant Condition $\times$ Region $[F(1,19)=2.80, p>.10, M S E=1.5]$, nor a Condition $\times$ Hemisphere $(F<1)$ interaction was found. The ANOVA performed on the three midline electrodes did not reveal either a significant main effect of condition $[F(1,19)=2.25, p>.10, M S E=11.9]$, or a 


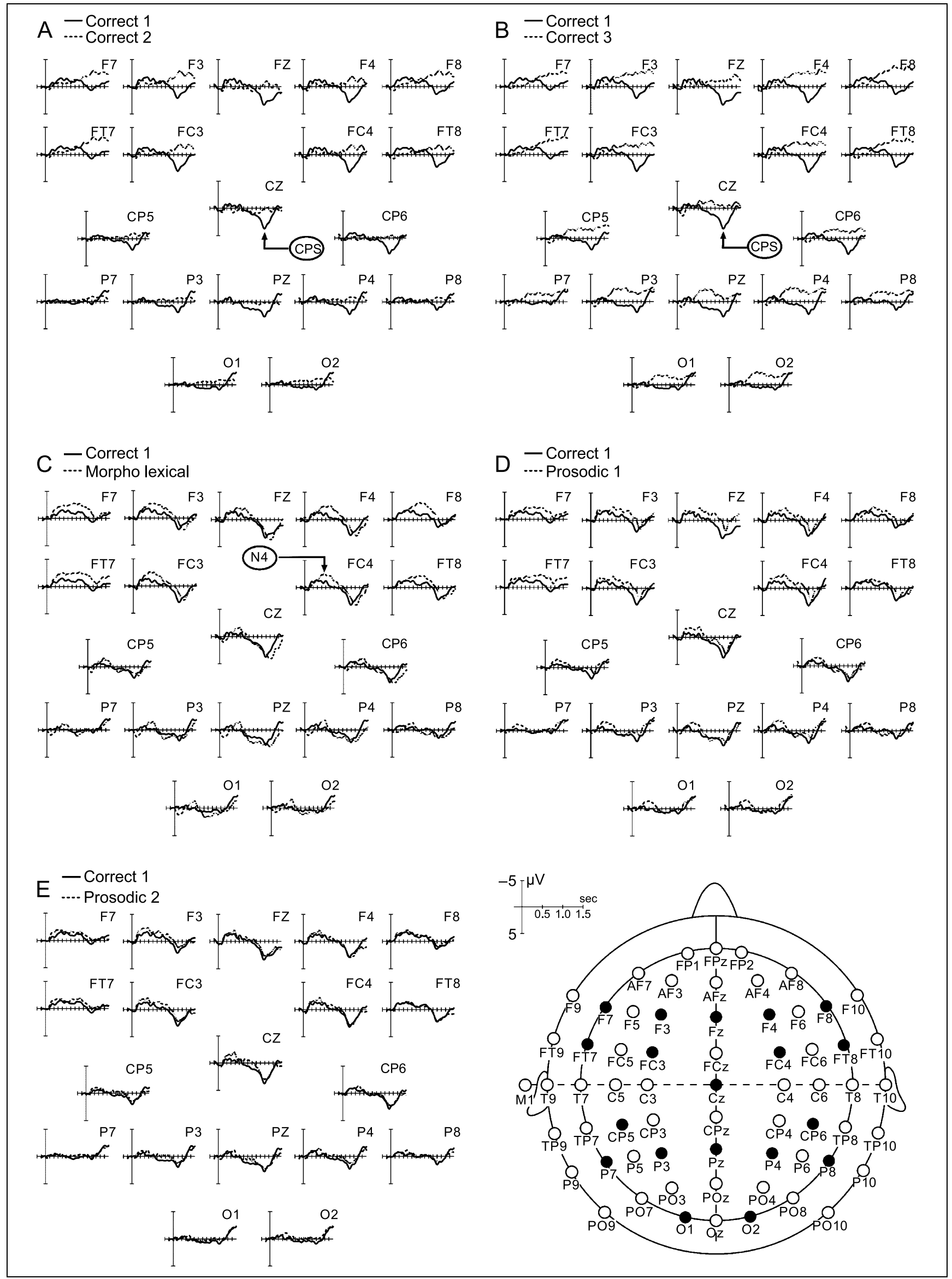


significant Condition $\times$ Electrode interaction $[F(2,38)=$ $1.52, p>.10, M S E=1.1]$.

As the critical tests to investigate the predictive power of prosody was given by the comparisons between (1C) and (1D), a subsequent three-way ANOVA with condition (3 levels: Correct 1, Morpholexical, Prosodic 1), region (anterior, posterior), and hemisphere (left, right) as within-subjects factors for the time window 300$500 \mathrm{msec}$ was run. A significant main effect of condition was found $[F(2,38)=3.50, p<.05, M S E=5.1]$, indicating that the amplitudes of the waveforms in the Correct 1, Morpholexical, and Prosodic 1 conditions differed in some way. Subsequent analyses revealed that the Morpholexical condition significantly differed from the Correct 1 condition $[F(1,19)=7.28, p<.05, M S E=4.2]$, reflecting an N400 effect. In contrast, conditions Prosodic 1 and Correct 1 did not significantly differ $[F(2,38)=$ $2.91, p>.10, M S E=7.8]$, indicating the absence of an N400 effect. Finally, the Morpholexical and Prosodic 1 conditions differed from each other. This difference was more pronounced in the anterior region than in the posterior one as indicated by the Condition $\times$ Region interaction $[F(1,19)=3.87, p=.06, M S E=2.3]$. Finally, the ANOVA performed on the three midline electrodes failed to reveal either a significant main effect of condition $[F(2,38)=2.50, p=.10, M S E=7.6]$, or a significant Condition $\times$ Electrode interaction $[F(4,76)=1.26, p>$ $10, M S E=1.6]$.

The ANOVA conducted for the time window 700 1000 msec revealed a significant Condition $\times$ Region interaction in the Morpholexical condition $[F(1,19)=$ $13.52, p<.01, M S E=1.8]$ as well as in the Prosodic 1 condition [1c; $F(1,19)=5.40, p<.05, M S E=2.2]$. Subsequent post hoc analyses showed that this interaction was due to a negativity in the anterior region for both the Morpholexical $[1 \mathrm{C} ; F(1,19)=7.04, p<$ $.05, M S E=1.7]$ and the Prosodic $1[1 \mathrm{D} ; F(1,19)=$ 5.40, $p<.05, M S E=1.1]$ conditions. No significant ERP effect was reported in the posterior region. Furthermore, the ANOVA run on the three midline electrodes only showed a marginally significant Condition $\times$ Electrode interaction in both the Morpholexical (1C) and Prosodic 1 (1D) conditions $[F(2,38)=3.06, p<$ $.10, M S E=1.4$ and $F(2,38)=2.74, p<.10, M S E=$ 2.3 , respectively].

The statistic analysis conducted on the late time window 1000-1500 msec revealed a significant difference between the Correct 2 and the Correct 1 conditions [1A; $F(1,19)=8.27, p<.01, M S E=13.1]$ as well as between the Correct 3 and the Correct 1 condition $[1 \mathrm{~B} ; F(1,19)=$ 42.91, $p<.01, M S E=5.7]$ comparisons. This effect reflects the larger positive shift (CPS) observed in the Correct 1 than in both the Correct 2 and Correct 3 conditions. Moreover, a significant Condition $\times$ Region interaction was found for the Correct 2/Correct 1 comparison $[1 \mathrm{~A} ; F(1,19)=15.68, p<.01, M S E=2.2]$, indicating that the CPS was larger in the anterior $[F(1,19)=17.21, p<.01, M S E=3.8]$ than in the posterior region $[F(1,19)=1.37, p>.10, M S E=3.8]$. The significant Condition $\times$ Region interaction revealed for the Correct 3/Correct 1 comparison [1B; $F(1,19)=$ $11.68, p<.01, M S E=1.9]$ reflects a larger CPS in the anterior region $[F(1,19)=57.87, p<.01, M S E=1.8]$ than in the posterior one $[F(1,19)=14.68, p<.01$ $M S E=2.0]$. Furthermore, a significant Condition $\times$ Region interaction was found in the Morpholexical condition $[F(1,19)=5.12, p<.05, M S E=1.0]$. However, subsequent post hoc analyses failed to show a significant effect of condition neither in the anterior $(F<1)$ nor in the posterior region $[F(1,19)=1.13, p>.10, M S E=2.3]$.

The ANOVA performed on the three midline electrodes in the late time window indicated a significant difference between the Correct 3 and the Correct 1 conditions $[1 \mathrm{~B} ; F(1,19)=15.33, p<.01 M S E=11.5]$ as well as a significant Condition $\times$ Electrode interaction $[F(2,38)=3.88, p<.05, M S E=2.5]$. This interaction indicates that the difference between the waveforms in the Correct 3 and Correct 1 conditions varied across the three $\mathrm{Fz}, \mathrm{Cz}$, and Pz electrodes. Post hoc tests revealed that the CPS was larger at the frontal electrode $\mathrm{Fz}$ $[F(1,19)=23.43, p<.01, M S E=5.3]$ than at the $\mathrm{Cz}$ electrode $[F(1,19)=9.13, p<.01, M S E=4.9]$; the CPS was only marginally significant $[F(1,19)=4.22, p<.10$, $M S E=6.2]$ at the Pz electrode. Moreover, a significant Condition $\times$ Electrode interaction was found for the Correct 2/Correct 1 comparison $[1 \mathrm{~A} ; F(1,19)=3.49, p<$ $.05, M S E=2.4]$. This interaction was due to the presence of a CPS at the Fz electrode site only $[F(1,19)=$ 3.49, $p<.05, M S E=2.4]$.

\section{DISCUSSION}

The aim of the present study was to examine whether the prosody of an inflected verb stem can inform the parser about the presence of a split particle at a later position in the sentence. For studying the present functional characteristic of prosody, we recorded ERPs elicited by the processing of split particles positioned at the end of spoken German sentences. Participants heard sentences containing inflected verbs whose prosodic contour marked the presence of a split particle although these verbs do not take one. Our results provide a clearcut picture: Prosody of inflected verb stems does assist the parser to predict whether it is necessary to wait for a split particle. An N400 effect was only found in the Morpholexical condition for the

Figure 1. Grand average ERPs for the Correct 1 condition (solid line) compared to the Correct 2 (A), the Correct 3 (B), the Morpholexical (C), the Prosodic 1 (D), and the Prosodic 2 (E) conditions (dotted line). Negative voltage is plotted up. 
processing of a particle occurring after a verb whose prosodic contour does signal the presence of a particle although it does not take one. The distribution of the N400 component found in the present research was similar to earlier studies on lexical integration in the auditory domain (Holcomb \& Neville, 1991). No N400 component, by contrast, was recorded for the processing of the same sentences when the prosodic contour of the inflected verbs did not mark the presence of a split particle (Prosodic 1 condition; see Figure 2$).{ }^{9}$ We therefore concluded that the prosodic contour of German inflected verbs may inform the parser whether or not the grammatical category of the sentence final word is a particle. This ability for predicting upcoming information probably allows the parser to more effectively compute on-line a representation of the phrase structure.

In addition, a late frontal negativity was found for both Morpholexical and Prosodic 1 conditions. This late negativity may reflect a checking procedure for attempting to combine together the particle and the verb. Furthermore, no P600 component was found for the processing of grammatically "illegal" split particles in either the Morpholexical or Prosodic 1 conditions. Finally, as previously reported in German by Steinhauer, et al. (1999), we replicated a CPS effect for the processing of critical items located at intonational phrase boundaries. The replication of the CPS using other sentence materials demonstrated the robustness of this ERP component.

\section{The N400 as an Indicator of Lexical Search Costs}

The N400 was the first ERP component identified by Kutas and Hillyard (1980) in correlation with postlexical

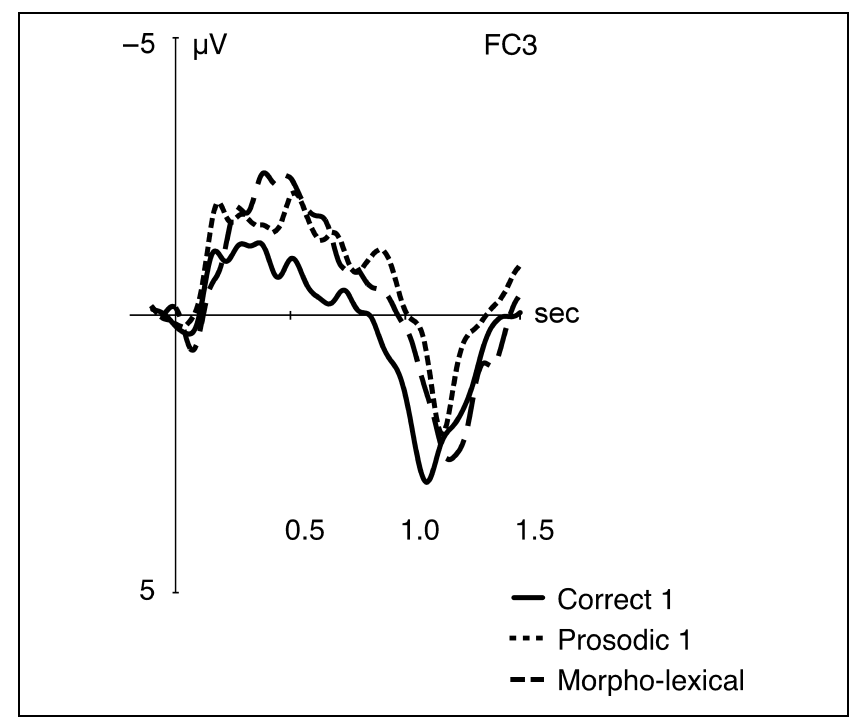

Figure 2. Grand average ERPs for the Correct 1, the Morpholexical, and the Prosodic 1 conditions at electrode FC3. An N400 component is elicited for the morpholexically incorrect sentences only. processes involved in lexical-semantic integration. In their pioneering work, Kutas and Hillyard showed a negativity $400 \mathrm{msec}$ post onset of a semantically anomalous sentence final word which was visually presented. As the N400 effect is usually reported to reflect difficulties in lexical-semantic integration (Chwilla, Brown, \& Hagoort, 1995), the question arises as to what cognitive processes are reflected in the N400 effect we measured for the processing of morpholexically incorrect sentences. Recently, N400 effects were observed for aspects other than purely lexical-semantic aspects of processing. Frisch and Schlesewsky (2001) observed an $\mathrm{N} 400$ for difficulties in thematic role assignment, for instance, when two animate arguments carry the same case marker. Based on these data, the N400 is taken to also reflect problems in thematic interpretation. Hopf, Bayer, Bader, and Meng (1998) reported an N400 for the processing of morphosyntactic violations such as pure case ambiguities. Hopf et al. (1998) interpreted the N400 component in their data as an indicator of a reaccess to the lexicon for assigning the appropriate case to the NP.

Here we propose that the N400 effect found in the Morpholexical condition reflects the costs associated with the lexical search which is initiated once the processing system attempts to activate the lexical code corresponding to the combination of the split particle and the verb stem. In accordance with the stem-affix model proposed by Marslen-Wilson, Tyler, Waksler, and Older (1994), we assume that the lexical entry of German verb forms like anlaecheln (to smile at) consists of the verb stem \{laecheln; (to smile) and a link to the split particle $\{a n ;$ (at) $\}$. The recognition of the verb form anlaecheln thus involves the access to the verb stem and to the associated particle. In German, split particles are obligatorily positioned at the end of the clause due to morphosyntactic constraints (see Note 1). Consequently, the processing of German verbs that take a split particle "inevitably" necessitates a (re)-access to the lexicon once the split particle is encountered. This "late" reaccess allows the language processing system to activate the appropriate verb. In the Morpholexical condition of the present study, a similar procedure might also be engaged. The language processor, however, failed to activate a lexical form as the combination of the verb stem and the split particle results in a pseudoverb, not stored in the mental lexicon. We assume that the lexical search in this case might be more difficult, and consequently, take more time. This assumption is supported by the accuracy data showing a high rate of errors (3.9\%) in the Morpholexical condition. Here we argue that the N400 effect in the present data reflects the costs associated with the lexical search, which is more difficult to be performed when the language processor is confronted with pseudoverbs. This interpretation is congruent with the extensive research on the N400 which showed that the processing of 
pronounceable nonwords (pseudowords) elicits a large N400 (Bentin, 1987). Moreover, our interpretation of the N400 component in terms of costs associated with the lexical search rather than in terms of lexical reaccess is also supported by the absence of an N400 effect in the Correct 2 condition despite the lexical reaccess obligatorily performed in processing the legal split particles.

Finally, to account for the absence of an N400 in the Prosodic 1 condition, despite the presence of a split particle after an inflected verb which does not take a particle, we propose that the language processor did not process the particle. The reason for this is that the prosodic information of the inflected verb did not mark the presence of a particle. Thus, having already achieved a successful lexical-semantic integration as well as a correct syntactic computation on the basis of the words preceding the particle, the language processor does not attempt to process additional linguistic elements, such as split particles not included in the predicted syntactic structure. On the basis of the present data, we propose that the language processor does not integrate prosodically nonpredicted words during a lexical integration, especially when short and without a prominent acoustic saliency as the split particles in our study.

In addition, we found a late frontal negativity for both Morpholexical and Prosodic 1 conditions. This late negativity may be due to a late checking procedure in order to check for a possible combination of the particle and the verb. This late checking process appears to be independent of prosodic information as the effect is observed for Morpholexical and Prosodic 1 conditions.

\section{Why Did the Processing of "Illegal" Split Particles Not Trigger Repair Processes?}

Repair of an ungrammatical syntactic structure is assumed to be reflected by a posteriorly distributed P600 (Kaan \& Swaab, 2003). A posterior P600 has been reported for the processing of sentences containing phrase structure violations (Friederici, Hahne, \& Mecklinger, 1996; Neville, Nicol, Barss, Forster, \& Garrett, 1991). In the Morpholexical condition of the present study, a split particle occurring after an inflected verb whose lexical entry does not contain a split particle inevitably creates a structural incongruity which results from the inability to attach the particle into the syntactic tree. Here, it is important to note that, due to the prosodic information of the inflected verbs marking the presence of an upcoming split particle in the Morpholexical condition, the structural incongruity can only be detected during a later stage of processing once the language processor has failed to successfully activate a verb in the mental lexicon. We assume that the impossibility to combine the split particle with the verb stem informs the language processor that the grammatical category of the last word may not be a particle. This information with respect to the grammatical category is probably represented at the levels of word form and morphological computation (Shapiro \& Caramazza, 2003). Consequently, the language processor might attempt to assign a new syntactic category (i.e., preposition) to the nonattachable element. The syntactic repair should be attested by a posterior P600. However, our ERP data did not reveal a P600 effect. To account for the absence of a P600 component, we consider the following explanation. As the prosodic information of the particle marked an intonational phrase boundary which indicates ending of sentence, no attempt for assigning another alternative word's grammatical category like a preposition might have been done, as prepositions are head of phrases and cannot be marked by an acoustic-prosodic information of closure.

\section{A New Feature in the Gee and Grosjean-type Binary Prediction System}

Taken together, the present findings provide strong support for the argument that intonation can be used very early during the processing of German sentences, that is, at the inflected verb in order to assist the parser to predict whether a split particle is coming up at the end of the sentence. Interestingly, the determining role of prosody to predict upcoming information has already been shown for the auditory processing of monomorphemic words in English (Davis, Marslen-Wilson, \& Gaskell, 2002) as well as for the processing of compounds in German (Isel, Gunter, \& Friederici, 2003). Isel et al. (2003) and Davis et al. (2002) showed that the duration of a monosyllabic word is crucial in determining whether this word is part of a longer word or not. With respect to the functional role of prosody to anticipate upcoming information, our data are in line with the prediction system proposed by Gee and Grosjean (1983). However, our findings shed new light on the conclusions one can draw concerning the position of the word in the sentence at which prosody can be proficient in order to anticipate the information that serves as input to the language processor. Grosjean and Grosjean and Hirt (1996) showed that the predictive power of prosody for estimating whether or not a sentence ends can only come into play when participants hear the potentially last word of the sentence (i.e., at a late position in the sentence). Here we demonstrated that prosody plays a crucial role early in the sentence (i.e., at the inflected verb stem) for signaling whether a split particle will be present at the end of sentences or not. Thus, on the basis of the ERP data we collected on the auditory processing of German split particles, we suggest that it might be fruitful to implement a new feature in the Gee and Grosjean-type prediction system, namely, $[+/-$ split particle]. We are 
fully aware, however, that it would be necessary to replicate the present experiment in different languages with the particular characteristics of split particles before to be able to validate this feature.

\section{Conclusions}

Drawing on the present electrophysiological evidence, we argue that prosody has a predictive power for "signaling" the language processor as to what information is coming up. More precisely, we clearly showed that prosody (1) helps predict linguistic elements that are located "far away" from the word containing the determinant prosodic information, and (2) comes into play early during the processing of the sentence. This finding with respect to the role of prosody in German might help to further specify the prediction system proposed by Gee and Grosjean (1983). We propose that it could be relevant to integrate into this prediction system a new feature, namely, $[+/-$ split particle], to adequately describe some languages.

\section{METHODS}

\section{Participants}

Twenty students of the University of Leipzig (12 men; 20-26 years; mean age 23.0 years) participated as paid volunteers in the study. All participants were native speakers of German, had normal or corrected-to-normal vision, had no known hearing deficit, and were righthanded (laterality quotient range $67-100 \%$ according to the Edinburgh handedness scale; Oldfield, 1971).

\section{Materials}

A total of 120 correct German sentences with an inflected verb whose lexical entry contained either a split particle such as an [at] for the verb laechelte an [smiled at] (Correct 1 and Correct 2 conditions, with 40 sentences in each condition), or no split particle as for the verb nannte [called] (Correct 3 condition consisting of 40 sentences) were constructed according to the format presented in Table 1 . These inflected verbs were words of high frequency (192 occurrences per million, $S E M=52.7$ for the verbs with particles; 257 occurrences per million, $S E M=67.8$ for the verbs without particles). A Morpholexical condition was derived from the Correct 3 condition by placing an illegal split particle at the end of the sentences, creating thus a morpholexical violation. ${ }^{10}$

The sentences in the Morpholexical condition were pronounced with a contour of intonation which was comparable with morpholexically correct sentences. However, post hoc acoustic measures showed that the production latencies of the inflected verb stems were significantly shorter in the Morpholexical condition
(758 msec, SEM $=27 \mathrm{msec}$ ) than in the Correct 3 condition ( $877 \mathrm{msec}$, SEM $=28 \mathrm{msec}, p<.01$ ). The 40 sentences of Prosodic 1 condition was constructed by cross-splicing $^{11}$ the first part of the Correct 13 condition consisting of the subject and the inflected verb, and the second part of the Morpholexical condition consisting of the direct object and the split particle, resulting in morpholexically and prosodically incorrect sentences. Finally, Prosodic 2 condition was derived by crosssplicing the first part of the Correct 2 condition (i.e., the subject and the inflected verb), and the second part of the Correct 1 condition (i.e., the direct object and the split particle). The cross-splicing manipulation of both Prosodic 1 and Prosodic 2 conditions is illustrated in Table 1 by means of different colors. On average, the sentences had 13 words. The split particles served as critical word in Correct 1, Morpholexical, Prosodic 1, and Prosodic 2 conditions, whereas the prepositions served as critical word in the Correct 2 and Correct 3 conditions. Furthermore, 240 visual probes were selected. A total of 120 probes were verbs or substantives presented in the spoken sentences. Mean frequency of these probes was 69 occurrences per million $(S E M=$ 10.5). On average, they had 7 letters. These 120 probes consisted of 40 verbs occupying the initial position in the sentences and 80 substantives occupying either the median $(n=40)$ or the final position $(n=40)$ in the sentences. For example, participants heard the sentence Sie laechelte den Arbeiter an und empfabl die Absicherung der neuen Grube [She smiled at the worker, and recommended the protection of the new ditch] and had to make a PDT either on the probe laechelte [smiled; initial position], Arbeiter [worker; median position], or Grube [ditch; final position].

Processing of these probes required a "yes" response. In addition, 120 visual probes, which were not part of the spoken sentences, were also used for balancing the number of yes and no responses over the experimental design. These probes were 40 verbs and 80 substantives which were matched on number of letters (on average, 7 letters) and frequency (54 occurrences per million, $S E M=11.1$ ) with the set of other 120 probes. For example, after having heard the sentence Sie laechelte den Arbeiter an und empfabl die Absicherung der neuen Grube, participants saw either the probe prablte [braged], or Roman [roman]. These probes required a "no" response.

The sentences were produced by a woman native speaker of standard German and recorded in a soundproof chamber. The digitized speech signals ( $44 \mathrm{kHz} /$ 16 bit sampling rate) of each sentence were measured with respect to word duration, and fundamental frequency (onset, peak, and offset) for the 38 inflected verbs and split particles (see Figure 3).

For the inflected verbs, a one-way ANOVA with the factor condition (Correct 1, Correct 2, Correct 3, Morpholexical) run on duration showed a significant main effect 
Figure 3. Duration (msec) and F0 (Hz) for the inflected verbs and for the split particles.

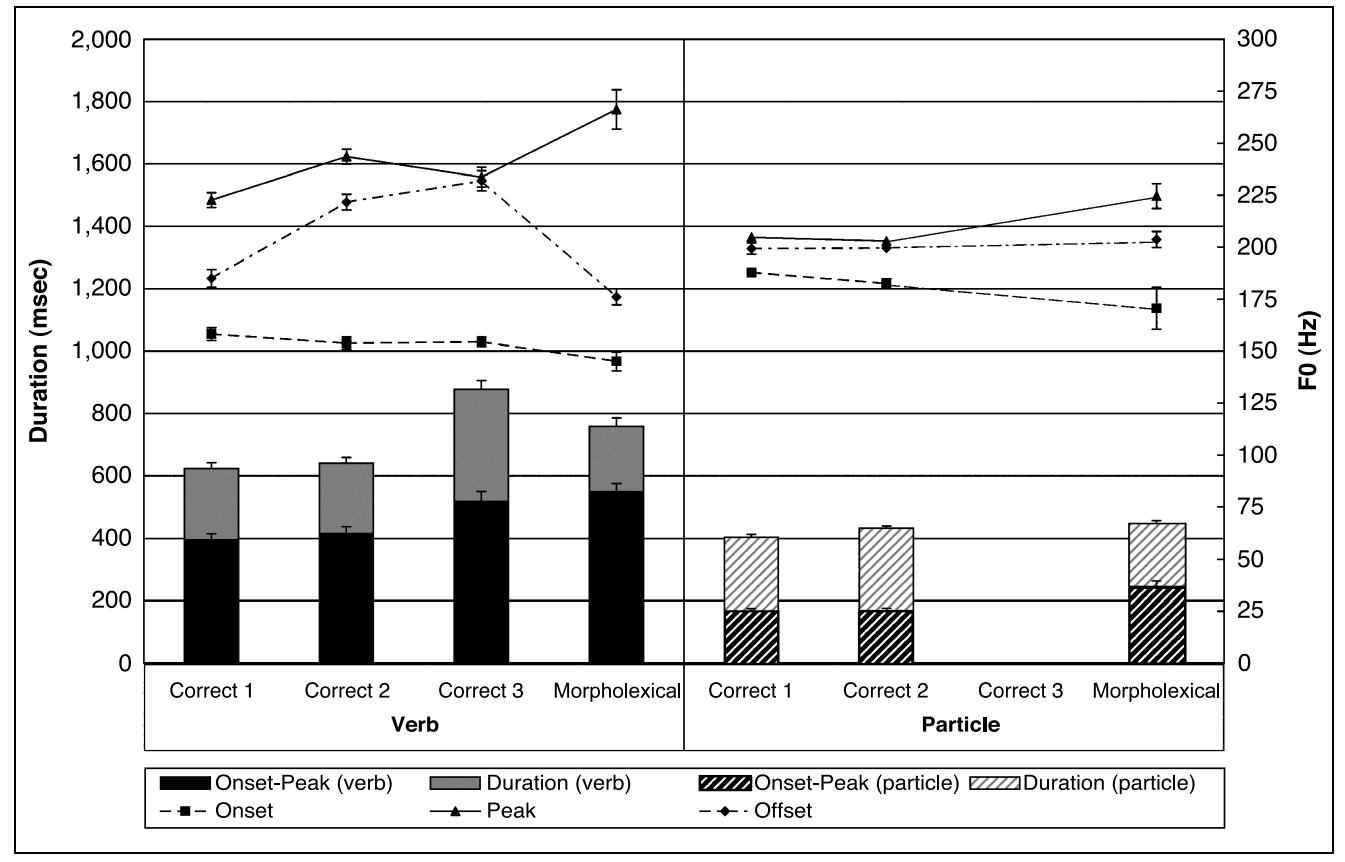

of condition $\left[F_{2}(3,111)=28.59, p<.01, M S E=\right.$ 18,509.3]. Further post hoc tests indicated that all pairwise comparisons were significant at $p<.01$, except for the Correct $1 /$ Correct 2 comparison $(p=.5)$. Moreover, a two-way ANOVA with the factors condition (4 levels), and F0 location (onset, peak, offset) run on F0 value revealed a significant main effect of both condition $\left[F_{2}(3,111)=8.14, p<.01, M S E=1103.3\right]$ and $\mathrm{F} 0$ location $\left[F_{2}(2,74)=417.80, p<.01, M S E=717.3\right]$, as well as a significant Condition $\times$ F0 location interaction $\left[F_{2}(6,222)=27.16, p<.01, M S E=621.8\right]$. Further post hoc analyses indicated that F0 onset was significantly lower in the Morpholexical condition than in the Correct 1 condition $(p<.05)$ but was only marginally lower than in both the Correct 2 and Correct 3 conditions $(p<.10)$. F0 peak was significantly higher in the Morpholexical than in the Correct 1 and Correct 3 conditions $(p<.01)$ as well as in the Correct 2 condition $(p<.05)$. Finally, F0 offset was significantly lower in the Morpholexical condition than in both the Correct 2 and Correct 3 conditions $(p<.01)$ but was only marginally lower than in the Correct 1 condition $(p<.10)$. A further one-way ANOVA with condition (4 levels) conducted on the duration between the F0 onset and the F0 peak revealed a significant main effect of condition $\left[F_{2}(3,111)=8.56, p<.01, M S E=25,347.3\right]$. Subsequent post hoc tests showed that mean duration in the Morpholexical condition was significantly longer than in both the Correct 1 and Correct 2 conditions. In contrast, Morpholexical and Correct 3 conditions did not differ significantly (see Figure 3).

For the split particles, the one-way ANOVA with the factor condition (Correct 1, Correct 2, Morpholexical) run on duration indicated a significant main effect of condition $\left[F_{2}(2,74)=5.94, p<.01, M S E=3084.4\right]$. Further post hoc tests indicated that particles in the Correct 1 conditions were significantly shorter than in both the Morpholexical $(p<.01)$ and Correct 2 conditions $(p<.05)$. Moreover, Correct 2 and Morpholexical conditions did not significantly differ $(p=0.3)$. An additional two-way ANOVA run on F0 value with the factors condition (3 levels) and F0 location (onset, peak, offset) revealed a significant main effect of F0 location $\left[F_{2}(2,74)=46.14, p<.01, M S E=588.4\right]$, as well as a significant Condition $\times$ F0 location interaction $\left[F_{2}(4,148)=7.12, p<.01, M S E=560.3\right]$. Further post hoc tests indicated that F0 onset in the Morpholexical condition was only marginally lower than in the Correct 1 condition. F0 peak in the Morpholexical condition was significantly higher than in the Correct 1 and in the Correct 2 conditions $(p<.01)$. Finally, F0 offset did not significantly differ across the three conditions $(p>3)$. A subsequent one-way ANOVA with the factor condition (3 levels) run on the duration between F0 onset and F0 peak indicated a significant main effect of condition $\left[F_{2}(2,74)=12.86, p<.01, M S E=\right.$ 6008.2]. Further post hoc analyses revealed that duration in the Morpholexical condition was significantly longer $(245 \mathrm{msec})$ than in both the Correct $1(167 \mathrm{msec})$ and Correct 2 conditions (168 msec, $p<.01)$. In contrast, Correct 1 and Correct 2 conditions did not differ significantly. In sum, on the basis of the present inferential statistics, the following acoustic-prosodic signature of the inflected verbs in the morpholexical condition emerges: longer duration, lower F0 onset and offset, and higher F0 peak usually observed with a long delay after the F0 onset. In contrast, no prominent acousticprosodic specificities were noticed for the split particles. 


\section{Procedure}

The 240 sentences (120 correct and 120 incorrect) were intermixed in 4 blocks of 60 sentences (10 sentences of each experimental condition). The 4 blocks were permuted in 4 lists of presentation such that each block appeared in each position of presentation (i.e., Position 1, 2, 3, and 4). Each of the lists was administered to a total of 5 participants. In each block, the sentences were presented in a pseudorandomized order with the following constraints. First, sentences from the same condition were presented in no more than three consecutive trials. Second, no more than three sentenceprobe pairs requiring either a "yes" or a "no" response were presented in succession. Finally, the lag between repetition of the same critical verb was 15 trials.

Participants were comfortably seated in a chair approximately $100 \mathrm{~cm}$ in front of a computer screen. They listened to the sentences via loudspeakers. The structure of each trial was as follows: A fixation star appeared at the center of a computer monitor $500 \mathrm{msec}$ before the auditory sentence presentation started, and remained visible until the end of the sentence. Then, the fixation star disappeared, and the visual probe was displayed in uppercase letters for $200 \mathrm{msec}$. Participants were allowed $3000 \mathrm{msec}$, from the time of probe presentation, in which to respond. Participants were asked to indicate by pressing one of two buttons, whether the visual probe was heard in the preceding sentence (PDT). The next trial started after an interstimulus interval (ISI) of $1000 \mathrm{msec}$. Timeout was set at 200 and $1500 \mathrm{msec}$; if the participants responded before $200 \mathrm{msec}$ or after $1500 \mathrm{msec}$, the response was coded as missing. Participants were instructed before the beginning of the experiment to avoid blinking and other movements during the presentation of the fixation star. The whole experiment lasted approximately $2 \mathrm{hr}$.

\section{EEG Recording}

Electroencephalogram (EEG) was continuously recorded (A/D at a rate of $250 \mathrm{~Hz})$ from 19 cap-mounted tin electrodes (midline: Fz, Cz, Pz; lateral: F7/8, F3/4, FT7/8, FC3/4, CP5/6, P7/8, P3/4, 0112) while subjects listened to the sentence in an electromagnetically shielded chamber. Additional electrodes were placed on the left and right outer canthus for recording horizontal electrooculogram (EOG), and above and below the right eye for vertical EOG measurements. All electrodes were referenced against the left mastoid. Impedances were kept below $5 \mathrm{k} \Omega$.

\section{Analysis}

Two sentences were eliminated in the Correct 3 condition due to erroneous codifications of the expected "yes" or "no" responses by the experimenter. For keeping a balanced design with 38 sentences in each condition, we also suppressed two sentences in the other five conditions.

\section{Behavioral data}

Mean percentages of errors and mean verification latencies to accurate responses were analyzed in two one-way repeated-measures ANOVAs with condition (6 levels) as within-subjects factor. If the main effect of condition was significant, pairwise comparisons were then conducted. Our policy regarding statistical reports is as follows: $F_{1}$ and $F_{2}$ are always computed, if appropriate. We consider an effect significant if both $F_{1}$ and $F_{2}$ meet the .05 criterion. For easier reading, we report $F_{1}$ only.

\section{EEG Analysis}

Only the correctly answered trials were analyzed. Epochs comprised the $200 \mathrm{msec}$ preceding and $1500 \mathrm{msec}$ following the critical item. Trials with excessive ocular artifacts or other movement artifacts were excluded from further analysis. The rejected trials due to artifacts were distributed equally over the six conditions with a mean of $14.26 \%(S E M=2.2)$ for the Correct 1 condition, $13.72 \%(S E M=2.0)$ for the Correct 2 condition, $11.65 \%(S E M=1.8)$ for the Correct 3 condition, $12.73 \%(S E M=2.2)$ for the Morpholexical condition, $10.39 \%(S E M=2.0)$ for the Prosodic 1 condition, and $13.98 \%$ (SEM $=2.9$ ) for the Prosodic 2 condition. The ANOVA run on the percentage of rejected trials with condition (6 levels) as within-subjects factor did not reveal a main effect of condition $(p=.20)$. ERPs were quantified as the mean amplitude relative to a 100-msec prestimulus baseline, using the following latency windows: 150-280 msec (ELAN), 300-700 msec (N400), 700-1000 msec (P600), 1000-1500 msec (CPS; see Note 8 ), based on the literature and the visual inspection of the grand averages. Each condition was evaluated separately against the Correct 1 condition.

The midline data were analyzed with a two-way repeated-measures ANOVA with the factors condition (6 levels) and electrode (Fz, Cz, Pz). The analyses for the lateral electrodes included two topographical variables which were completely crossed: region (anterior, posterior), and hemisphere (left, right). Four quadrants resulted from this crossing: left anterior (F7, F3, FT7, FC3), right anterior (F8, F4, FT8, FC4), left posterior (CP5, P7, P3, O1), and right posterior (CP6, P8, P4, O2). For effects involving more than one degree of freedom, the Greenhouse-Geisser correction (Greenhouse \& Geisser, 1959) was applied, to avoid Type I errors due to unequal variances between the conditions. Post hoc pairwise comparisons at single electrode sites were performed using a modified Bonferroni procedure with $\alpha$ set to .05 (Keppel, 1991). 


\section{Acknowledgments}

This work was supported by grants awarded to F. I. by the University of Leipzig (Zentrum fuer Kognitionwissenschaften) and by the German Research Foundation (DFG IS 76/1-2) as well as by the Human Frontier Science Program (HFSP RGP5300/2002-C102) awarded to K. A. We thank Silke Urban who made available to us the sentence materials she employed in her dissertation. We are also grateful to Shirley-Ann Rueschemeyer for helpful comments on an initial version of the manuscript. K. A. is now working at the University of Newcastle, UK.

Reprint requests should be sent to Frédéric Isel, Max Planck Institute for Human Cognitive and Brain Sciences, PO Box 500 355, 04303 Leipzig, Germany, or via e-mail: isel@cbs.mpg.de.

\section{Notes}

1. Although in German split particles are obligatorily positioned at the end of the clause (e.g., Se laechelte den Arbeiter an [She smiled the worker at]), in English they are directly placed after the verbal phrase (e.g., She smiled at the worker).

2. Note, however, that listeners' ability to resolve global ambiguity seems to vary depending on whether speakers were aware of the possibility of multiple interpretations when they produced the sentence.

3. In the diagnosis model proposed by Fodor and Inoue (1994), the disambiguating element is called the symptom. The symptom, more or less transparent, is the first word of an input string that cannot be attached at all, or at least not sensibly into the current tree. Although this word marks an error, this word does not show what the error was.

4. In this paradigm, a spoken language stimulus is presented in segments of increasing duration (i.e., the gates) and participants are asked to propose the word being presented and to give a confidence rating after each segment.

5. A point to be noted is that prediction is quite random at the beginning of the potentially last word, whereas it is very good at the end.

6. However, the amplitude of the CPS is smaller for visual than for auditory processing.

7. The literal translation in English is "She smiled the worker $a t^{\prime \prime}$.

8. A statistic analysis was also conducted for a time window 800-1500 msec. Similar results were reported as for the time window 1000-1500 msec. Here we choose to use a time window 1000-1500 msec for investigating the CPS. We ensured thus to avoid a temporal overlap with the time window 700-1000 msec used for studying the P600 effect.

9. The difference between the acoustic-prosodic patterns of the verb stems in the Morpholexical condition (i.e., short duration) and in the Prosodic 1 condition (i.e., long duration) might be reflected by a wide distributed negativity. The ERPs plotted at the onset of verb stems suggest that the verb stems with a long duration (i.e., Prosodic condition) elicited a larger negativity than the verb stems with a short duration (i.e., Morpholexical condition) in a time window 300-700 msec. This finding reinforces the idea that prosody of the verb stems in the Morpholexical condition sufficiently contrasted with prosody in the Prosodic 1 condition in order to assist the parser for anticipating the occurrence of a split particle at the end of the sentence.

10. A yes/no paper-and-pencil pretest of lexical judgment was conducted with 24 participants in order to ensure that the 40 verbs used in the Morpholexical condition were judged as pseudoverbs in German. Participants were instructed to read
240 particle-verb pairs in the infinitive form and to decide whether each pair constitutes or not a German verb. Only the verbs which were judged in 100\% of cases as pseudoverbs were kept for the present experiment.

11. An amplitude normalization was applied for each sentence in order to protect against detectability of the signal manipulation at the splicing point.

\section{REFERENCES}

Beach, C. M. (1991). The interpretation of prosodic patterns at points of syntactic structure ambiguity: Evidence for cue trading relations. Journal of Memory and Language, 30, 644-663.

Bentin, S. (1987). Event-related potentials, semantic processes, and expectancy factors in word recognition. Brain and Language, 31, 308-327.

Chwilla, D. J., Brown, C., \& Hagoort, P. (1995). The N400 as a function of the level of processing. Psychophysiology, 32, 274-285.

Cutler, A., Dahan, D., \& van Donselaar, W. (1997). Prosody in the comprehension of spoken language: A literature review. Language and Speech, 40, 141-201.

Davis, M. H., Marslen-Wilson, W. D., \& Gaskell, M. G. (2002). Leading up the lexical garden path: Segmentation and ambiguity in spoken word recognition. Journal of Experimental Psychology: Human Perception and Performance, 28, 218-244.

Ferreira, F., Anes, M. D., \& Horine, M. D. (1996). Exploring the use of prosody during language comprehension using the auditory moving window technique. Journal of Psycholinguistic Research, 25, 273-290.

Fodor, J. D. (2002). Psycholinguistics cannot escape prosody. In B. Bel \& I. Marlien (Eds.), Proceedings of the 1st International Conference on Speech Prosody (pp. 83-88). Keikichi Hirose Laboratory, University of Tokyo, Tokyo, Japan.

Fodor, J. D., \& Inoue, A. (1994). The diagnosis and cure of garden path. Journal of Psycholinguistic Research, 23, 407-434.

Frazier, L. (1987). Sentence processing: A tutorial review. In Coltheart M. (Ed.), Attention and Performance XII (pp. 559-586). Hillsdale, NJ: Erlbaum.

Friederici, A. D. (1995). The time course of syntactic activation during language processing: A model based on neuropsychological and neurophysiological data. Brain and Language, 50, 259-281.

Friederici, A. D. (2002). Towards a neural basis of auditory sentence processing. Trends in Cognitive Sciences, 6, 78-84.

Friederici, A. D., \& Alter, K. (2004). Lateralization of auditory language functions: A dynamic dual pathway model. Brain and Language, 89, 267-276.

Friederici, A. D., Hahne, A., \& Mecklinger, A. (1996). Temporal structure of syntactic parsing: Early and late event-related brain potential effects elicited by syntactic anomalies. Journal of Experimental Psychology: Learning, Memory, and Cognition, 22, 1219-1248.

Frisch, S., \& Schlesewsky, M. (2001). The N400 reflects problems of thematic hierarchizing. NeuroReport, 12, 3391-3394.

Gee, J., \& Grosjean, F. (1983). Performance structures: A psycholinguistic and linguistic appraisal. Cognitive Psychology, 15, 411-458.

Gorrell, P. (1995). Syntax and parsing. Cambridge: Cambridge University Press.

Greenhouse, S., \& Geisser, S. (1959). On methods in the analysis of profile data. Psychometrica, 24, 95-112. 
Grosjean, F. (1983). How long is the sentence? Prediction and prosody in the on-line processing of language. Linguistics, 21, 501-529.

Grosjean, F., \& Hirt, C. (1996). Using prosody to predict the end of sentences in English and French: Normal and brain-damaged subjects. Language and Cognitive Processes, 11, 107-134.

Holcomb, P. J., \& Neville, H. J. (1991). Natural speech processing: An analysis using event-related brain potentials. Psychobiology, 19, 286-300.

Holmes, V. M., Kennedy, A., \& Murray, W. S. (1987). Syntactic structure and the garden path. Quarterly Journal of Experimental Psychology, 39A, 277-293.

Hopf, J.-M., Bayer, J., Bader, M., \& Meng, M. (1998). Event-related brain potentials and case information in syntactic ambiguities. Journal of Cognitive Neuroscience, 10, 264-280.

Isel, F., Gunter, T. C., \& Friederici, A. D. (2003). Prosody-assisted head-driven access to spoken German compounds. Journal of Experimental Psychology: Learning, Memory, and Cognition, 29, 277-288.

Kaan, E., \& Swaab, T. Y. (2003). Repair, revision, and complexity in syntactic analysis: An electrophysiological differentiation. Journal of Cognitive Neuroscience, 15, 98-110.

Keppel, G. (1991). Design and analysis (3rd ed.). Englewood Cliffs, NJ: Prentice-Hall.

Kutas, M., \& Hillyard, S. A. (1980). Reading senseless sentences: Brain potentials reflect semantic incongruity. Science, 207, 203-205.

Lehiste, I. (1972). The timing of utterances and linguistic boundaries. Journal of the Acoustical Society of America, 51, 2018-2024.

Lehiste, I. (1973). Phonetic disambiguation of syntactic ambiguity. Glossa, 7, 107-122.

Marslen-Wilson, W. D., \& Tyler, L. K. (1980). The temporal structure of spoken language understanding. Cognition, 8, $1-71$.

Marslen-Wilson, W. D., Tyler, L. K., Waksler, R., \& Older, L. (1994). Morphology and meaning in the English mental lexicon. Psychological Review, 101, 3-33.

Marslen-Wilson, W. D., Tyler, L. K., Warren, P., Grenier, P., \& Lee, C. S. (1992). Prosodic effects in minimal attachment. Quarterly Journal of Experimental Psychology, 45A, 73-87.

McClelland, J. L., St. John, M., \& Taraban, R. (1989). Sentence comprehension: A parallel distributed processing approach. Language and Cognitive Processes, 4, 287-336.

Nagel, H. N., Shapiro, L. P., Tuller, B., \& Nawy, R. (1996). Prosodic influences on the resolution of temporary ambiguity during on-line sentence processing. Journal of Psycholinguistic Research, 25, 319-344.

Neville, H. J., Nicol, J., Barss, A., Forster, K. I., \& Garrett, M. F. (1991). Syntactically based sentence processing classes: Evidence from event-related brain potentials. Journal of Cognitive Neuroscience, 3, 151-165.

Nicol, J. L., \& Pickering, M. J. (1993). Processing syntactically ambiguous sentences: Evidence from semantic priming. Journal of Psycholinguistic Research, 22, 207-237.

Oldfield, R. (1971). The assessment and analysis of handedness: The Edinburgh inventory. Neuropsychologia, 9, 97-113.

Price, P. J., Ostendorf, M., Shattuck-Hufnagel, S., \& Fong, C. (1991). The use of prosody in syntactic disambiguation.

Journal of the Acoustical Society of America, 90, 2956-2970.

Pynte, J., \& Prieur, B. (1996). Prosodic breaks and attachment decisions in sentence parsing. Language and Cognitive Processes, 11, 165-191.

Shapiro, K., \& Caramazza, A. (2003). The representation of grammatical categories in the brain. Trends in Cognitive Sciences, 7, 201-206.

Speer, S. R., Kjelgaard, M. M., \& Dobroth, K. M. (1996). The influence of prosodic structure on the resolution of temporary syntactic closure ambiguities. Journal of Psycholinguistic Research, 25, 249-271.

Steinhauer, K., Alter, K., \& Friederici, A. D. (1999). Brain potentials indicate immediate use of prosodic cues in natural speech processing. Nature Neuroscience, 2, 191-196.

Steinhauer, K., \& Friederici, A. D. (2001). Prosodic boundaries, comma rules, and brain responses: The closure positive shift in ERPs as a universal marker for prosodic phrasing in listeners and readers. Journal of Psycholinguistic Research, 30, 267-295.

Stirling, L., \& Wales, R. (1996). Does prosody support or direct sentence processing? Language and Cognitive Processes, 11, 193-212.

Warren, P., Grabe, E., \& Nolan, F. (1995). Prosody, phonology and parsing in closure ambiguities. Language and Cognitive Processes, 10, 457-486.

Watt, S. M., \& Murray, W. S. (1996). Prosodic form and parsing commitments. Journal of Psycholinguistic Research, 25, 291-318. 